\title{
Simultaneous and successive presentations of single-featured and multi-featured visual forms: Implications for the parallel processing hypothesis
}

\author{
STUART M. KEELEY` AND MICHAEL E. DOHERTY \\ BOWLING GREEN UNIVERSITY
}

\begin{abstract}
The parallel processing hypothesis predicts no difference in hit rate (HR) for identical forms presented simultaneously or successively. This was tested in two experiments differing only in the number of features distinguishing stimulus forms: (1) one feature (Landolt Cs); (2) multiple features (the graphemes A, T, U). Each experiment had three conditions: (1) single form, (2) four simultaneous forms, (3) four successive forms. The major finding was a lack of $H R$ increase for four simultaneous $C s$ over one $C$. HRs for successive Cs and both multiple letter conditions sharply increased over the one form condition. Results call into question the level at which parallel processing occurs. Three decision models, all assuming perceptual independence, were tested. None fit all the data.
\end{abstract}

A model assuming multiple independent opportunities to perceive has been proposed to describe the outcome of experiments in which the same form was presented a number of times simultaneously in a brief presentation (Eriksen \& Lappin, $1965 ; 1967)$ and where two different forms were presented successively, separated by intervals from 0 to 1500 msec (Eriksen, 1966). Such a model is based on a concept of perceptual independence, which assumes that at any point in time, the varying sensitivities in the visual perceptual system are uncorrelated for stimulus forms separated by some minimal distance on the fovea. It is further assumed that there is a lack of interaction between forms simultaneously presented on these separate foveal areas. In the framework of this model, the Eriksen and Lappin (1965) results suggested the possibility of at least six parallel channels in visual perception.

In the present experiment, four identical forms are presented both simultaneously and successively at an energy level sufficient for above chance identification of a single form but insufficient for $100 \%$ performance. A direct comparison between the hit rates (HRs) obtained for a like number of redundant stimuli presented under both simultaneous and successive conditions has implications for convergence on the nature of the processing of multiple briefly presented forms. If indeed the HR for multiple, simultaneously presented forms reflects multiple independent opportunities to perceive the form, HRs would not be expected to differ under the two conditions, providing the decision processes are similar, and providing the number of forms does not exceed the number of independent channels. Green and Swets (1966) cite several alternative models for describing how an observer arrives at an overall decision, given multiple independent observations. According to their decision-threshold model, each of several observations leads to a decision, and it is the decisions that are combined in making an overall decision. Under this model an increase in detectability results from multiple observations because each additional observation presents another detection opportunity. Alternatively, Green and Swets' integration model assumes that information from individual observations is added together to form the accumulation of evidence that is used to make an overall decision. The experimental procedure permits the testing of the appropriateness of these alternative models of the S's decision processes.

\section{EXPERIMENT 1}

Subjects

Three faculty members and three assistants served in this experiment.

\section{Apparatus}

A Scientific Prototype Model GB Tachistoscope with a hand switch permitting $S$ to initiate stimulus onset was used.

Stimuli

To have forms varying in only one dimension, Landolt rings (Cs) were employed. The original Cs were Letraset Sheet No. 116 "Os," rubbed onto a black background, with approximately $23 \mathrm{deg}$ of the circumference opaqued before photographing. The Cs were located at the $45 \mathrm{deg}, 135 \mathrm{deg}, 225 \mathrm{deg}$ and $315 \mathrm{deg}$ positions on an imaginary circle (considering the top to be $0 \mathrm{deg}$ ), centered on a fixation point. The circle subtended a visual angle of $1.8 \mathrm{deg}$, the Cs $.3 \mathrm{deg}$. The minimum separation between forms was $1.25 \mathrm{deg}$. A single form stimulus had one $C$ at one of the four loci, with the gap either right, left, up or down. Thus, there were 16 different single form stimuli. A simultaneous condition stimulus had four forms, a $\mathrm{C}$ at each of the four loci with all gaps in the same direction. There were four different, four-form stimuli. The luminance of the stimulus field was $.22 \mathrm{ft}-\mathrm{L}$, and when trans-illuminated, provided $99 \%$ contrast.

\section{Procedure}

All viewing was monocular, with S's preferred eye. Three conditions of stimulus presentation were employed. In all conditions $\mathrm{S}$ responded by naming the perceived direction of the gap, and by stating a confidence judgment indicating whether he "felt sure," "thought so" or was "guessing." These were announced by $S$ as " 1 ," "2" or " 3 ," respectively. Stimulus presentation was self-initiated by $S$ after a ready signal, which was the sound of the stimulus being inserted.

Single form $(1 \mathrm{C})$. One single-form stimulus per trial was presented with $S$ responding to each stimulus. All Ss were pretrained under this condition. Pretraining continued until an exposure duration yielding a stable IC HR between .40 and .60 was established. This duration was then held constant for a given $S$ across all conditions for the entire experiment. The exposure durations ranged from 3.75 to $4.75 \mathrm{msec}$.

Simultaneous ( $4 \mathrm{C})$. One four-form stimulus per trial was presented, with $\mathrm{S}$ responding to each stimulus.

Successive (IC4). Four single-form stimuli, with the gaps in the same direction but with the location of the Cs randomized, were presented in succession. The $S$ responded only after the fourth presentation. Since stimulus changing was manual, the interpresentation interval was several seconds. Randomization of gap direction was restricted in all three conditions such that there was an equal number of right, left, up and down gap trials within each block of 20.

An experimental session began with about $7 \mathrm{~min}$ of dark adaptation. The $\mathbf{S}$ was then given four practice presentations appropriate to his condition. He was instructed not to initiate presentation until the fixation point, which was on at all times, was in clear vision.

A given experimental session consisted of 40 stimulus presentations, either $101 \mathrm{C} 4$ trials or $201 \mathrm{C}$ and $204 \mathrm{C}$ trials. After pretraining each $S$ participated in 18 experimental sessions, 12 1C4 sessions and six sessions each consisting of $204 \mathrm{C}$ and $20 \mathrm{lC}$ trials. Thus, there were 120 trials in each condition. For half of the Ss, two IC4 sessions were run first, then one combined $4 \mathrm{C}$ and $1 \mathrm{C}$ session, then two $1 \mathrm{C} 4$, etc., until 18 sessions were completed. The other three Ss began with a combined session, then alternated 
Table 1

Obtained HRs for Each S for Each of the Three Experimental Conditions, and HRs Predicted by the Clearest Form, Integration and Availability Models

\begin{tabular}{lccccccc}
\hline $\mathrm{S}$ & IC & 4C & IC4 & $\begin{array}{c}\text { Clearest } \\
\text { Form }\end{array}$ & Integration & $\begin{array}{c}\text { Decision } \\
\text { Threshold }\end{array}$ \\
\hline 1 & .608 & .608 & .783 & .748 & .887 & .944 \\
2 & .600 & .525 & .758 & .740 & .880 & .940 \\
3 & .533 & .467 & .783 & .682 & .798 & .887 \\
4 & .517 & .475 & .675 & .568 & .778 & .871 \\
5 & .433 & .467 & .658 & .492 & .632 & .753 \\
6 & .425 & .458 & .692 & .507 & .617 & .740 \\
Av & .519 & .500 & .725 & .623 & .765 & .856 \\
\hline
\end{tabular}

with pairs of $1 \mathrm{C} 4$ sessions until all 18 were run. For half the combined sessions, the $1 \mathrm{C}$ condition was run first, in the other half the $4 \mathrm{C}$. No knowledge of results was given $\mathrm{S}$, except that he was told his number of hits after each block of 10 trials.

\section{Results}

Table 1 presents the empirical and predicted $\mathrm{HR}$ values for each $S$. Contrary to expectations, the mean $1 \mathrm{C}$ and $4 \mathrm{C}$ HRs do not differ; $\mathbf{t}(5)=.95$, n.s. In contrast, the mean $1 \mathrm{C} 4 \mathrm{HR}$ is significantly higher than both the $1 \mathrm{C} H R, t(5)=10.30, p<.01$, and the $4 \mathrm{C} \mathrm{HR,} \mathrm{t}(5)=10.97, \mathrm{p}<.01$.

The Eriksen (1966) model makes explicit the following assumptions underlying the prediction of HRs for multiple observations from single observation HRs: (1) observations are independent; (2) different confidence judgments (" 1 ," " 2 ," " 3 ") reflect different perceptual states ("A," "B," "C"); (3) if multiple stimulation results in different perceptual states, $S$ 's decision will be determined by the state represented by the most certain confidence judgment. Under these assumptions for the case in which there are four forms presented and three categories of confidence jedgments, step one in making the predictions involves estimating the probability of occurrence of each of the $3^{4}$ possible combinations of perceptual states. The empirical proportion of occurrence of a given confidence judgment in the $\mathrm{IC}$ condition is used to estimate the probability of occurrence of the respective perceptual state on any single observation of a multiple observation trial. Under assumption (3) above, the sum of the probabilities of occurrence of all combinations containing an $A$ state are multiplied by the A state 1C HR. Similarly the sum of the probability of occurrence of all combinations containing a $B$ state, but no A states is multiplied by the B state HR. Finally the probability of the one combination containing all $\mathrm{C}$ states is multiplied by the $\mathrm{C}$ state HR. The sum of these three products is the predicted HR.

Table 1 shows clearly that predictions based on this model do not fit the empirical data. The mean $4 \mathrm{C}$ HR differed from the predicted HR, $t(5)=3.79, p<.05$, with every S's HR being below the predicted HR. This contrasts with the results obtained by Eriksen and Lappin (1965), who found that obtained results tended to be greater than predicted results. The mean 1C4 HR differed from the predicted $H R, t(5)=3.75, p<.05$, with every S's HR being above that predicted.

Under the above model, an S's decision on any trial is determined solely by the observation associated with the clearest perceptual state. In the case of multiple occurrences of the clearest state, the predicted HR is no greater than the case of a single occurrence, e.g., the predicted HR associated with the occurrence of an AAAA combination (i.e., four A states) is no greater than for an ACCC combination. In fact, the A state HR is the upper bound for the predicted HR under this model, regardless of the actual number of independent opportunities to perceive.

\section{Decision-Threshold Model}

According to the decision-threshold model, an increase in detectability results from multiple observations, not because each observation represents an additional opportunity for a clearer perceptual state, but rather because each additional observation presents another independent detection opportunity. Under this model the HRs for all observations within a trial, rather than the
HR for the clearest observation, determine the prediction. Such a model has been elaborated by Green and Swets (1966).

For a forced-choice task, the assumption is made that $S$ will be correct on all repetitions on which the form produces a supra-threshold sensory measure (i.e., is "available"), and that $S$ will guess randomly on all other repetitions. Under these assumptions, the probability of $S$ being correct on a given trial, $\mathbf{P}(\mathrm{C})$, is

$$
P(C)=p+(1 / m)(1-p)
$$

where $\mathrm{p}$ is the probability of a form getting correctly through a perceptual channel, and $m$ is the number of response alternatives. The predicted HR $\left(H R^{\prime}\right)$ for a trial with $n$ repetitions of a form is

$$
H R^{\prime}=1-(1-p)^{n}+1 / m(1-p)^{n}
$$

where $p$ is estimated by substituting S's obtained $1 \mathrm{C} \mathrm{HR}$ for $\mathrm{P}(\mathrm{C})$ in Eq. 1.

Table 1 indicates that this model significantly overestimates the obtained $\mathrm{HRs}$ for both the $4 \mathrm{C}, \mathrm{t}(5)=13.69, \mathrm{p}<.01$, and $1 \mathrm{C} 4$, $t(5)=5.46, p<.01$, conditions.

\section{An Integration Model}

A fundamentally different alternative to the above two models is the integration model (Green \& Swets, 1966), which is predicated on the assumption that information, rather than decisions, from separate observations is accumulated, and it is the accumulated information which forms the basis for the decision. The case of the integration model tested here further assumes (1) observations are combined without information loss, (2) the observations are independent, and (3) each observation is normally distributed with the same variance under noise and signal plus noise. This set of assumptions permits the application of statistical decision theory, and the use of $\mathrm{d}^{\prime}$. The general formula for predicting a multiple observation HR from single observation data is

$$
d_{n}^{\prime}=\left(n d^{\prime 2}\right)^{1 / 2}
$$

where the $d^{\prime}$ for the 1C HR can be directly obtained from Swets (1964, Table 11, p. 682f). The $H^{\prime}$ can then be read directly from this table using the value of ${\mathrm{d}^{\prime}}_{n}$ calculated in Eq. 3 .

The predicted $\mathrm{HRs}$ for the $4 \mathrm{C}$ condition differ significantly from the obtained HRs, $t=8.39, \mathrm{df}=5, \mathrm{p}<.01$. However, this model fares somewhat better with predictions of the 1C4 data, $t=1.34, \mathrm{df}=5$, n.s., but this is by no means compelling evidence for the model (see Table 1).

\section{EXPERIMENT 2}

The most unexpected finding in Experiment 1 was the lack of any improvement in HR in the $4 \mathrm{C}$ condition beyond the $1 \mathrm{C} \mathrm{HR}$. Eriksen and Lappin (1965) found a marked increase in HR as the number of redundant stimuli presented simultaneously increased from 1 to 4 . The major difference between our $4 \mathrm{C}$ condition and their simultaneous condition is the type of form used. In our study, Landolt Cs, which vary only in a single dimension, the direction of a gap, were used. Eriksen and Lappin presented letters, which differ multidimensionally. Thus, one possible explanation for the discrepancy between the two studies is the type of form used. An increase in HR with multidimensional forms and not with unidimensional forms would have important theoretical implications for the question of serial vs parallel information processing. A second experiment was run using the same procedures as in Experiment 1, but with letters instead of Landolt Cs.

\section{Method}

Subjects 1 and 5 from Experiment 1 served in this experiment. The apparatus and procedures were the same as in Experiment 1, except as follows. The forms were the letters A, T and U (Letraset Sheet 441 ) which, when photographed, were approximately the 
Table 2

Obtained and Predicted HRs for Experiment 2

\begin{tabular}{lcccccc}
\hline S & SL & $4 \mathrm{~L}$ & SL4 & $\begin{array}{c}\text { Clearest } \\
\text { Form }\end{array}$ & Integration & $\begin{array}{c}\text { Decision } \\
\text { Threshold }\end{array}$ \\
\hline 1 & .560 & .677 & .726 & .660 & .780 & .873 \\
5 & .452 & .690 & .679 & .714 & .565 & .696 \\
Av & .506 & .684 & .702 & .687 & .672 & .784 \\
\hline
\end{tabular}

same size as the Cs. New exposure durations appropriate to single letter (1L) HRs between .40 and .60 were established by pretraining, and were 2.0 and $2.5 \mathrm{msec}$. The $1 \mathrm{~L}$ and $4 \mathrm{~L}$ conditions were run within the same session in blocks of 21 trials each, with seven presentations of each form. The 1L4 sessions were now paired on successive days such that 10 trials were run in one session, 11 in the other, with seven of each form. Four sessions of counterbalanced blocks of $1 \mathrm{~L}$ and $4 \mathrm{~L}$ trials, and eight of $1 \mathrm{~L} 4$ trials were run. Thus, there were 84 trials in each condition. The Ss responded $\mathrm{A}, \mathrm{T}$ or $\mathrm{U}$, with confidence judgments.

\section{Results}

Predictions of the several models are made exactly as in Experiment 1, since the several models do not consider the complexity or dimensionality of the single forms. The results, presented in Table 2, are radically different from those of Experiment 1 in several essential respects. It is clear, even with just two Ss, that the $4 \mathrm{~L}$ HR is not only greater than the IL HR, but is about the same as the 1L4 HR. It is difficult to assess the models with the Experiment 2 data, but it seems from the two Ss that the clearest form model would fare quite well, while the others would not.

\section{DISCUSSION}

The results of the above experiments are relevant to two important theoretical questions: (1) Is the processing of briefly presented multiple form displays parallel, reflecting multiple independent detection opportunities? (2) Given the situation in which $S$ has multiple independent opportunities to perceive a form, how does he combine the observations to arrive at an overall decision? It is important to make this distinction, since all of the proposed models are irrelevant for predicting simultaneous condition HRs if indeed parallel processing does not exist.

In Experiment 1, Landolt Cs were selected as display forms to obtain simultaneous and successive presentation. HRs for forms differing in a single, distinctive feature. When these unidimensional forms are used, four simultaneous presentations do not lead to an increase in HR beyond that obtained for a single form presentation. Four successive presentations do provide a significant increase. This lack of increase in the $4 \mathrm{C}$ condition, in combination with the systematic increase in the $1 \mathrm{C} 4$ condition, argues against the parallel processing hypothesis for these forms. The data suggest that $S$ does not get multiple independent opportunities to perceive in the $4 \mathrm{C}$ condition, and therefore a test of the model against the $4 \mathrm{C}$ data is not meaningful.

The lack of correspondence between our results and previous findings appears to be due primarily to a difference in the nature of the stimulus forms used. Even though results were obtained from only two Ss, Experiment 2 indicated that indeed when letters are presented simultaneously, our results correspond closely to previous findings (Eriksen \& Lappin, 1965). The Ss identify $4 \mathrm{~L}$ displays more accurately than $1 \mathrm{~L}$, and at a HR comparable to $1 \mathrm{L4}$. These results do support a parallel processing hypothesis.

This discrepancy of results in the two experiments calls into question the level at which parallel processing may occur. Since the simultaneous presentation HR does not increase with unidimensional forms and does increase with multidimensional forms, it appears likely that paralle] processing may be occurting at the level of features, rather than at the level of forms. This is compatible with the notion of Neisser (1967) that the process of recognition is hierarchically organized. For instance before $S$ decides $A$ is present in the input, he makes prior decisions about subordinate features, e.g., angles, surface area, diverging lines. The elementary operations to detect $A$ would be expected to be different from those detecting a $\mathrm{T}$ or $\mathrm{U}$. However, in the case of detecting the gap of a $\mathrm{C}$, there is only a single feature which will distinguish one (' from another, the direction of the gup. While there is insufficient data to propose a formal model at this time, there is a suggestion that the lack of HR increase in the $4 C$ condition reflects the fact that only one feature is available for analysis, irrespective of the number of forms. If, for example, a feature analyzer operates on a binary, present-absent basis, and if there is only one analyzer for a given feature, the single feature aspect of the $4 \mathrm{C}$ condition would obviate the possibility of any improvement. That is, any one feature analyzer gets one "look," and if it is in error. no correction is possible. On the other hand, in the $4 \mathrm{~L}$ condition, there is an unspecifiably large number of features available to different feature analyzers. The HR would be expected to increase with an increased number of forms: since the different forms would be expected to activate different analyzers, thereby providing opportunities for correction. Such an interpretation is compatible with the finding that increasing the dimensionality of forms has its greatest impact on the simultaneous presentation, since in the successive presentation, even a single feature analyzer would receive multiple "looks."

It seems that what is critically needed to resolve some of the questions raised here is research manipulating both number of forms presented and the dimensionality of the forms.

In terms of our second question concerning the decision processes of Ss given multiple opportunities to perceive, none of the models proposed provides a consistently good fit for the successive presentation data. Implicit in all three models is the assumption that S's errors are random. A close analysis of our $1 \mathrm{C4}$ and $1 \mathrm{L4}$ data indicates that errors are not random, and that Ss show strong biases in their non-correct judgments. Models typically assume that when $\mathrm{S}$ makes an error his decision is based on below threshold, or insufficient information, not on incorrect information. Both the empirical data and Ss' subjective reports indicate that Ss often perceive a stimulus clearly but erroneously, an error which might be labeled a "confusion" error. As a result of this type of finding, it is possible that the most appropriate model for predicting the successive presentation results is one which takes into account combinations of conditional probabilities and views the $\mathrm{S}$ as making his decision on the basis of likelihood ratios. For example, we can consider an $\mathbf{S}$ as a decision maker who has $\mathrm{n}$ independent pieces of information, $e_{1}, e_{2}, \ldots e_{n}$, all of which are relevant to a decision among $m$ hypotheses, $h_{1}, h_{2}, \ldots h_{m}$. The probability of each of the $m$ possible response hypotheses, given each of the possible combinations of events, can be computed according to Bayes theorem in the manner prescribed by Green and Swets $(1966$, p. 28). We can then assume that $S$ selects the response associated with the greatest conditional probability.

The advantage of such a model is that it takes into account all the information, including the nature of the $\mathrm{Ss}$ ' errors, in describing the decision making process. Data for testing such a model is now being collected.

\section{REFERENCES}

ERIKSEN, C. W. Independence of successive inputs and uncorrelated error in visual form perception. J. exp. Psychol., 1966, 72, 26-35.

ERIKSEN, C. W., \& LAPPIN, J. S. Independence in the perception of simultaneously presented forms at brief durations. J. exp. Psychol., 1967, $73,468-472$.

ERIKSEN, C. W., \& LAPPIN, J. S. Internal perceptual system noise and redundancy in simultaneous inputs in form identification. Psychon. Sci., $1965,2,351-352$.

GREEN, D. M., \& SWETS, J. A. Signal detection theory and psychophysics. New York: Wiley, 1966.

NĖISSER, U. Cognitive psychologv. New York: Appleton-Century, 1967.

SWETS, J. A. (Ed.) Signal detection and recognition by human observers: Contemporary readings. New York: Wiley, 1964.

\section{NOTE}

1. Address: Department of Psychology, Bowling Green State University, Bowling Green, Ohio 43402.

(Accepted for publication June 13, 1968.) 